\title{
PRÉ-DESPACHO HIDROTÉRMICO DE POTÊNCIA ATIVA E REATIVA VIA MÉTODO DOS PONTOS INTERIORES E COORDENADAS RETANGULARES
}

\author{
Saimon S. Borges* \\ Katia Campos de Almeida ${ }^{\dagger}$ \\ *Centro Politécnico - DELT-TC-UFPR \\ Caixa Postal - 19011 \\ CEP 81531-980 - Curitiba PR \\ ${ }^{\dagger}$ GSP-Labspot-UFSC \\ 88040-900, Florianópolis, SC
}

Thelma S. Piazza Fernandes*

\begin{abstract}
Hydro-thermal scheduling of active e reactive power generation using rectangular form and Interior Points Method

This article presents the mathematical formulation of the active and reactive power generation pre-dispatch problem, whose objective function is the minimization of thermal plant costs and transmission losses. Bus voltages are represented in rectangular coordinates, transmission limits are imposed through linearized constraints and the optimization problem is solved by the Primal Dual Interior Point Method. The efficiency of the formulation is analyzed in a 5 buses example and an equivalent of the Paraná system composed of 291 buses.
\end{abstract}

Keywords: Interior Points Method, Pre-Dispatch Problem, Cartesian Coordinates

Artigo submetido em 05/07/2010 (Id.: 01166)

Revisado em 25/10/2010, 17/03/2011

Aceito sob recomendação do Editor Associado Prof. Julio Cesar Stacchini Souza

\section{RESUMO}

Este artigo apresenta a formulação matemática de um problema de pré-despacho de geração de potência ativa e reativa no qual a função objetivo engloba minimização dos custos de usinas térmicas e minimização das perdas elétricas na transmissão. A representação do fasor tensão é feita em coordenadas retangulares, consideram-se os limites de transmissão utilizando a formulação linearizada e o problema de otimização é resolvido pelo Método de Pontos Interiores versão Primal-Dual. A eficiência da formulação é testada utilizando-se o sistema de 5 barras e o sistema de 291 barras que representa o sistema elétrico reduzido do estado do Paraná.

Palavras Chaves: Método de Pontos Interiores, PréDespacho de Geração Hidrotérmica, Coordenadas Cartesianas

\section{INTRODUÇÃO}

Os sistemas hidrotérmicos de geração são compostos de usinas termelétricas e hidrelétricas convencionais e não convencionais ligadas aos centros de carga através de um sistema de transmissão. Na operação desse sistema, busca-se determi- 
nar uma estratégia de geração de cada usina que minimize o valor esperado dos custos operativos no horizonte de planejamento.

A disponibilidade de energia hidráulica, na forma do volume de água armazenado nos reservatórios, faz com que o problema de gestão destas reservas torne-se complexo, pois há uma relação entre as decisões operativas tomadas num estágio qualquer e suas consequiências futuras. Por outro lado, a impossibilidade de se conhecer perfeitamente as afluências futuras dos aproveitamentos hidroelétricos e a curva de carga do sistema torna sua operação um problema probabilístico. Por fim, a existência de várias bacias interligadas e a necessidade de avaliação do uso das reservas de água nos anos futuros levam ao emprego de um período longo de estudo, caracterizando o planejamento da operação como um problema de grande porte, que deve levar em conta um amplo espectro de atividades, abrangendo desde a otimização plurianual dos reservatórios até o despacho das usinas.

Assim, requer-se a utilização de diferentes horizontes de estudo, que correspondem a diferentes tipos de análises do desempenho do sistema, tais como: efeitos de longo prazo (possibilidade de déficits futuros, valor esperado de gerações térmicas no futuro e outros), efeitos de médio prazo (contratos anuais para suprimento de energia e demanda entre empresas do sistema, programação de manutenção futura e outros) e efeitos de curto prazo (controle de cheias, restrições de segurança futuras e outros). Ou seja, as complexidades do problema de operação não podem ser acomodadas por um modelo matemático único e torna-se necessária a utilização de cadeias de modelos com diferentes horizontes de planejamento e graus de detalhes.

A cadeia de planejamento do sistema hidrotérmico brasileiro, por exemplo, inclui: Planejamento da Operação de Médio Prazo (dois a cinco anos à frente), Planejamento de Curto Prazo (um ano à frente), Planejamento de Diário ou PréDespacho (um dia à frente) e Operação em Tempo Real (uma a meia hora à frente).

Atualmente, no Brasil, quando da verificação da segurança operativa do sistema, análises de fluxo de carga são realizadas considerando as potências ativas fornecidas pelas usinas fixadas nos valores definidos pelo pré-despacho. No entanto, estudos visando determinar a capacidade de transferência entre subsistemas indicam que o sistema é muito melhor aproveitado se é feito o re-despacho de potência ativa para maximizar a transferência de potência (Almeida et alii, 2009). Se a representação completa da parte elétrica for feita nos modelos de pré-despacho, essas alterações nas potências ativas das usinas não seriam necessárias e um melhor aproveitamento do sistema seria possível.
Assim sendo, este trabalho, que se concentra na etapa de PréDespacho, apresenta uma formulação do problema de otimização relacionado a essa etapa, onde tanto a parte ativa quanto a parte reativa do problema são modeladas, assim como as restrições de magnitude de tensão e carregamento das linhas. O modelo enfatiza a programação de geração e intercâmbio de energia elétrica em intervalos horários para o próximo dia, levando em conta os horizontes de planejamento anteriores, aspectos relativos à economia e segurança operacional do sistema elétrico.

A modelagem e o desenvolvimento de algoritmos para resolver o problema de pré-despacho de geração tomaram grande impulso a partir da década de 1960, com modelos baseados em equações de coordenação (Drake et alii, 1962). Na década de 1970, surgiram modelos mais sofisticados que consideravam restrições de transmissão (Saga e Khapade, 1978). Na década de 1980, foram muito exploradas técnicas onde os aspectos elétricos foram inseridos no planejamento de curtíssimo prazo, destacando-se os trabalhos de Habibollahzadeh e Bubenko (1986), Luo et alii (1989), Pereira e Pinto (1983), Carvalho e Soares (1987), Carvalho et alii (1988) que utilizaram programação baseada em fluxo em redes e/ou Fluxo de Potência Ótimo modelo linear (FPOCC) para a resolução do despacho de geração. A partir de 1990, observou-se um crescente detalhamento na modelagem do problema, sendo este resolvido por diferentes métodos. Ohishi (1990) propõe uma metodologia baseada em uma técnica híbrida usando otimização e simulação. Franco et alii (1994) resolvem o problema, que considera restrições de acoplamento hidráulico entre usinas e limites de transmissão, através de decomposição e técnica de penalidade linear-quadrática. Soares e Salmazo (1997) estudaram as perdas verificadas na operação de uma usina hidrelétrica e propuseram sua minimização como critério de despacho.

A representação do modelo não linear da rede elétrica foi introduzida no problema no final dos anos 1990 (Wei et alii, 1998). Os trabalhos de Nepomuceno e colaboradores, (Nepomuceno et alii, 2000 e 2002) propuseram uma formulação do problema sem representação das restrições hidráulicas, que incorpora restrições associadas à parte reativa do sistema de transmissão. Mais recentemente, variáveis discretas representando o status de ligado/desligado das unidades geradoras e restrições associadas foram introduzidas no problema, tendo sido empregadas diferentes técnicas na sua resolução (Conejo e Redondo, 1999; Sifuentes e Vargas, 2007; Lu et alii, 2010).

Assim, levando em consideração: (i) as experiências de Nepomuceno et alii (2000 e 2002), que ignoram as restrições hidráulicas a fim de simplificar o problema de PD e viabilizar a modelagem tanto da parte de potência ativa quanto a potência reativa e, (ii) o desempenho do método Primal Dual de Pon- 
tos Interiores (PDPI) na resolução do problema estático formulado em coordenadas retangulares por Torres e Quintana (1998) e Thomaz (2007), propõe-se neste trabalho formular o problema de pré-despacho representando as equações de balanço e potência e reativa na forma retangular e o resolvendo pelo PDPI. Deve-se destacar que a escolha da forma retangular apresenta as vantagens de contornar questões numéricas, pois as restrições do problema se tornam quadráticas e, conseqüentemente, mais fáceis de trabalhar. Outra vantagem é que a matriz Hessiana do problema é constante e a expansão em Taylor é exata para o termo de ordem dois.

A disposição deste trabalho é dada como se segue: inicialmente apresenta-se o problema de Pré-Despacho (PD). Na sequiência, é apresentada a formulação matemática do problema e, finalmente, é feita a apresentação de resultados e a conclusão.

\section{PRÉ-DESPACHO}

O Pré-Despacho (PD) de um sistema hidrotérmico é um modelo de planejamento cujo objetivo é o cálculo de uma política de geração de para o próximo dia e em base horária, para as unidades geradoras de um sistema de energia. O cálculo desta política de geração é feito de modo que certos critérios operacionais (mínimas perdas, mínimas complementação térmica, etc.) sejam otimizados e que determinadas restrições operativas (atendimento da demanda, limites nas gerações, nos fluxos de potência nas linhas, etc.) sejam atendidas.

Em sistemas fortemente hidráulicos como o brasileiro, o problema de PD está inserido em uma cadeia de planejamento energético, envolvendo modelos de longo, médio e curto prazo. Nesses sistemas a solução fornecida pelo PD deve levar em conta ainda as metas energéticas estabelecidas por modelos de médio prazo.

A inclusão das restrições de metas energéticas torna o PD um problema dinâmico, ou seja, decisões de geração em um determinado intervalo são dependentes das gerações calculadas para os demais intervalos do dia.

O problema pode ser visto como um agrupamento de problemas de fluxo de potência ótimo (FPO), um para cada período de tempo, cujas soluções devem atender também restrições intertemporais, buscando otimização dos recursos hídricos e térmicos disponíveis, visando ganhos econômicos.

No modelo brasileiro, pode-se considerar ainda, que as usinas hidráulicas têm potência suficiente para alimentar a carga, mas não dispõem de energia suficiente (água nos reservatórios) para atender a carga durante todo o horizonte de tempo. Segundo Wood e Wollenberg (1998), nessa situ- ação de programação hidrotérmica com restrição de energia, a usina hidrelétrica pode alimentar toda a carga apenas em alguns intervalos do horizonte de operação, havendo necessidade de complementação térmica nos outros intervalos de tempo. A maneira de se gerar a potência térmica ao longo do horizonte de operação da térmica é de forma constante durante o período que se faça necessário.

Como a tomada de carga dos geradores não é instantânea, ou seja, ela se dá sob a forma de uma rampa cuja inclinação é ditada pelos limites físicos das unidades geradoras, há de se considerar restrições intertemporais que estabeleçam inter-relação entre variáveis associadas a diversos intervalos de tempo do horizonte de operação considerado para as térmicas.

Como a inclusão dessas restrições na formulação do PréDespacho representa uma extensão não trivial dos algoritmos existentes, resolveu-se evitar a consideração de tomada de carga das térmicas, conforme Nepomuceno et alii (2000), realizando o despacho das térmicas de forma constante ao longo de todo o período de operação.

Os casos estudados nesse trabalho se encaixam na situação em que a potência instalada é maior que a demanda. Assim, a geração térmica é despachada com potência constante e pode não ser necessária durante todo o intervalo de tempo (Wood e Wollenberg, 1998). A fim de evitar as complicações da inclusão da tomada de carga, adotou-se o procedimento sugerido por Nepomuceno et alii (2000) e simplificou-se o problema, despachando-se a térmica por todo o intervalo de tempo

Assim, este trabalho formula um modelo de pré-despacho de potência ativa e reativa para sistemas hidrotérmicos que determina uma estratégia de geração que minimize o valor dos custos operativos e perdas elétricas no horizonte de 1 dia , com as seguintes considerações:

- Planejamento diário (24 horas) de geração de potência ativa e reativa;

- Utilização de metas energéticas obtidas por modelo de médio prazo;

- Restrições hidráulicas ignoradas, pois se supõe que os reservatórios variam muito pouco de armazenamento nesse horizonte de 24 horas (Nepomuceno et alii, 2000);

- Despacho das térmicas realizado de forma constante ao longo do dia (Nepomuceno et alii, 2000).

- Monitoração de reserva girante obtida por modelo de médio prazo (Provençano, 2003);

- Monitoração dos limites de fluxo de potência ativa adotando-se a formulação do fluxo de potência lineari- 
zado, simplificando a monitoração dessa grandeza (Provençano, 2003).

Salienta-se que o diferencial desse trabalho consiste em utilizar o método PDPI num modelo de pré-despacho com representação AC da rede elétrica e que considera o fasor tensão na forma retangular.

\section{FORMULAÇÃO DO PRÉ-DESPACHO}

Primeiramente, serão descritos os dados de entrada para resolução do PD.

\subsection{Dados de Entrada}

O primeiro dado de entrada é referente à carga que deve ser atendida ao longo das 24 horas e representada pelo vetor $\mathbf{P d}$ (demanda de potência ativa) e o vetor Qd (demanda de potência reativa) ambos com dimensão $[(n b . n p) \times 1]$ sendo $n b$ o número de barras e $n p$ o número de períodos:

$$
\mathbf{P d}=\left[\begin{array}{lllllll}
P d_{1}^{1} & \ldots & P d_{n b}^{1} & \ldots & P d_{1}^{n p} & \ldots & P d_{n b}^{n p}
\end{array}\right]^{t}
$$

$$
\mathbf{Q d}=\left[\begin{array}{lllllll}
Q d_{1}^{1} & \ldots & Q d_{n b}^{1} & \ldots & Q d_{1}^{n p} & \ldots & Q d_{n b}^{n p}
\end{array}\right]^{t}
$$

onde $P d_{i}^{k}$ representa a demanda de potência ativa na barra $i$ no período $k$ e $Q d_{i}^{k}$ representa a demanda de potência reativa na barra $i$ no período $k$.

Também é classificado como variável de entrada o vetor Meta de metas energéticas para as usinas hidráulicas, fornecida pelo modelo de médio prazo dado como:

$$
\text { Meta }=\left[\begin{array}{lll}
M e t a_{1} & \ldots & \text { Meta } \\
\text { nb }
\end{array}\right]^{t}
$$

onde $M$ et $a_{i}$ representa a meta energética para a usina hidráulica localizada na barra $i$, para o período total considerado.

Outro dado de entrada é o vetor referente à reserva girante, $\mathbf{R}$, para as usinas selecionadas pelos modelos otimização de médio prazo:

$$
\mathbf{R}=\left[\begin{array}{lllllll}
R_{1}^{1} & \ldots & R_{n b}^{1} & \ldots & R_{1}^{n p} & \ldots & R_{n b}^{n p}
\end{array}\right]^{t}
$$

onde $R_{i}^{k}$ representa a reserva de geração ativa para a usina localizada na barra $i$ período $k$.

A reserva girante corresponde a uma restrição de segurança e pode ser entendida como a capacidade de complementação mínima de produção de um conjunto determinado de usinas. A inclusão deste tipo de restrição impõe uma limitação na produção de um subconjunto das usinas presentes em um sistema (Provençano, 2003).

Os próximos dados de entrada referem-se aos limites máximos e mínimos de geração de potência ativa e reativa dos geradores térmicos e hidráulicos:

$$
\text { Ptermax }=\left[\begin{array}{lll}
\text { Ptermax }_{1} & \ldots & \text { Ptermax }_{n b}
\end{array}\right]^{t}
$$

onde Ptermax $_{i}$ representa o limite de potência ativa máximo de uma usina térmica localizada na barra $i$;

$$
\text { Phidmax }=\left[\begin{array}{lll}
\operatorname{Phidmax}_{1} & \ldots & \operatorname{Phidmax}_{n b}
\end{array}\right]^{t}
$$

onde $P$ hidmax ${ }_{i}$ representa o limite de potência ativa máximo de uma usina hidráulica localizada na barra $i$;

$$
\text { Qtermax }=\left[\begin{array}{lll}
\text { Qtermax }_{1} & \ldots & \text { Qtermax }_{n b}
\end{array}\right]^{t}
$$

onde Qtermax ${ }_{i}$ representa o limite potência reativa máximo de uma usina térmica localizada na barra $i$;

$$
\text { Qhidmax }=\left[\begin{array}{lll}
\operatorname{Qhidmax}_{1} & \ldots & \text { Qhidmax }_{n b}
\end{array}\right]^{t}
$$

onde $\operatorname{Qhidmax}_{i}$ representa o limite de potência reativa máximo de uma usina hidráulica localizada na barra $i$.

Conforme o número de períodos analisado, esses vetores se repetem sequencialmente a fim de ficarem com a mesma dimensão dos vetores Pd e Qd.

$$
\operatorname{Ptmax}=\left[\begin{array}{lll}
\text { Ptermax } & \text { Ptermax }
\end{array}\right]^{t}
$$

$$
\begin{gathered}
\text { Phmax }=\left[\begin{array}{lll}
\text { Phidmax } & \ldots & \text { Phidmax }
\end{array}\right]^{t} \\
\text { Qtmax }=\left[\begin{array}{lll}
\text { Qtermax } & \ldots & \text { Qtermax }
\end{array}\right]^{t}
\end{gathered}
$$

$$
\text { Qhmax }=\left[\begin{array}{lll}
\text { Qhidmax } & \text { Q } & \text { Qhidmax }
\end{array}\right]^{t}
$$

Existem relações semelhantes para limites mínimos de geração de potência ativa e reativa para térmicas e hidráulicas: Ptmin, Phmin, Qtmin, Qhmin.

A seguir, são descritas as variáveis de otimização envolvidas no PD. 


\subsection{Variáveis de Otimização}

O fasor tensão é representado na forma retangular, como usado em (Fernandes, 2004):

$$
\left[\begin{array}{l}
\dot{V}_{1}^{1} \\
\vdots \\
\dot{V}_{n b}^{1} \\
\ddots \\
\dot{V}_{1}^{1} \\
\vdots \\
\dot{V}_{n b}^{n p}
\end{array}\right]=\left[\begin{array}{l}
e_{1}^{1} \\
\vdots \\
e_{n b}^{1} \\
\ddots \\
e_{1}^{1} \\
\vdots \\
e_{n b}^{n p}
\end{array}\right]+j\left[\begin{array}{l}
f_{1}^{1} \\
\vdots \\
f_{n b}^{1} \\
\ddots \\
f_{1}^{1} \\
\vdots \\
f_{n b}^{n p}
\end{array}\right]
$$

onde $\dot{V}_{i}^{k}$ representa o fasor tensão na barra $i$ no período $k$, $e_{i}^{k}$ representa a parte real do fasor $\dot{V}_{i}^{k}$ e $f_{i}^{k}$ representa a parte imaginária do fasor .

A variável de otimização relacionada à geração de potência ativa pelas usinas hidrelétricas é o vetor Pgh:

$$
\mathbf{P g h}=\left[\begin{array}{lllllll}
P h_{1}^{1} & \ldots & P h_{n b}^{1} & \ldots & P h_{1}^{n p} & \ldots & P h_{n b}^{n p}
\end{array}\right]^{t}
$$

onde $P h_{i}^{k}$ é a geração de potência ativa na barra $i$ no período $k$.

Na modelagem deste trabalho, optou-se por considerar a potência ativa das usinas térmicas constante para todos os períodos, desta forma, vetor $\mathbf{P t}$ (geração ativa térmica) é repetido $n p$ vezes a fim de representar Pgt:

$$
\text { Pgt }=\left[\begin{array}{lllllll}
P t_{1} & \ldots & P t_{n b} & \ldots & P t_{1} & \ldots & P t_{n b}
\end{array}\right]^{t}
$$

onde $P t_{i}$ é a geração de potência ativa da usina térmica localizada na barra $i$.

A soma vetorial da geração e potência ativa das usinas hidráulica e térmica fornece a potência ativa total gerada:

$$
\mathbf{P g}=\mathbf{P g h}+\mathbf{P g t}
$$

A variável de otimização relacionada à geração de potência reativa pelas usinas hidrelétricas é o vetor Qgh e pelas usinas térmicas é o vetor Qgt (geração térmica):

$$
\mathbf{Q g h}=\left[\begin{array}{lllllll}
Q h_{1}^{1} & \ldots & Q h_{n b}^{1} & \ldots & Q h_{1}^{n p} & \ldots & Q h_{n b}^{n p}
\end{array}\right]^{t}
$$

onde $Q h_{i}^{k}$ é a geração de potência reativa na barra $i$ no período $k$.
$\mathbf{Q g t}=\left[\begin{array}{lllllll}Q t_{1}^{1} & \ldots & Q t_{n b}^{1} & \ldots & Q t_{1}^{n p} & \ldots & Q t_{n b}^{n p}\end{array}\right]^{t}$

onde $Q t_{i}^{k}$ é a geração de potência reativa na barra i no período $\mathrm{k}$.

A soma vetorial da geração e potência reativa das usinas hidráulica e térmica fornece a potência ativa total gerada:

$$
\mathrm{Qg}=\mathrm{Qgh}+\mathrm{Qgt}
$$

\subsection{Critério de Otimização}

A função objetivo do problema é minimização da função custo de geração de usinas térmicas e perdas elétricas:

$$
\text { f.o. }=w c \cdot \mathbf{c}(\mathbf{P g t})+w p \cdot \sum(\mathbf{P g t}+\mathbf{P g h})
$$

onde $w c$ é o coeficiente ajustável relacionado aos custos de geração térmica, $w p$ é o coeficiente ajustável relacionado às perdas elétricas e c(Pgt) é a função custo da geração térmica. Deve-se notar que ao se minimizar o segundo termo de (20), são minimizadas as perdas de transmissão em cada intervalo de tempo.

\subsection{Restrições de Igualdade}

\subsubsection{Equações de Balanço de Potência Ativa e Reativa}

O vetor de potências injetadas para todos os períodos e todas as barras é:

$$
\dot{\mathbf{S}}=\mathbf{P}+j \mathbf{Q}=\mathbf{P g}-\mathbf{P d}+j(\mathbf{Q g}-\mathbf{Q d})
$$

onde

$\dot{\mathbf{S}}$ : vetor de injeção de potência aparente englobando todos os períodos, dimensão $[n b . n p \times 1]$;

P: vetor de injeção de potência ativa englobando todos os períodos, dimensão $[n b . n p \times 1]$;

Q: vetor de injeção de potência reativa englobando todos os períodos, dimensão $[n b . n p \times 1]$.

Utilizando a definição de injeção de potência tem-se: 


$$
\begin{aligned}
& \dot{\mathbf{S}}=\left[\begin{array}{ccccccc}
\dot{V}_{1}^{1} & & & & & & \\
& \ddots & & & & & \\
& & \dot{V}_{n b}^{1} & & & & \\
& & & \ddots & & & \\
& & & & \dot{V}_{1}^{n p} & & \\
& & & & & \ddots & \\
& & & & & & \dot{V}_{n b}^{n p}
\end{array}\right] \text {. } \\
& \cdot\left[\begin{array}{ccc}
\dot{\mathbf{Y}}^{*} & & \\
& & \\
& \ddots & \\
& & \\
& & \dot{\mathbf{Y}}^{*}
\end{array}\right]\left[\begin{array}{c}
\dot{V}_{1}^{1} \\
\vdots \\
\dot{V}_{n b}^{1} \\
\vdots \\
\dot{V}_{1}^{n p} \\
\vdots \\
\dot{V}_{n b}^{n p}
\end{array}\right]
\end{aligned}
$$

Separando-se a parte real e parte imaginária da equação (22) obtem-se (Fernandes, 2004):
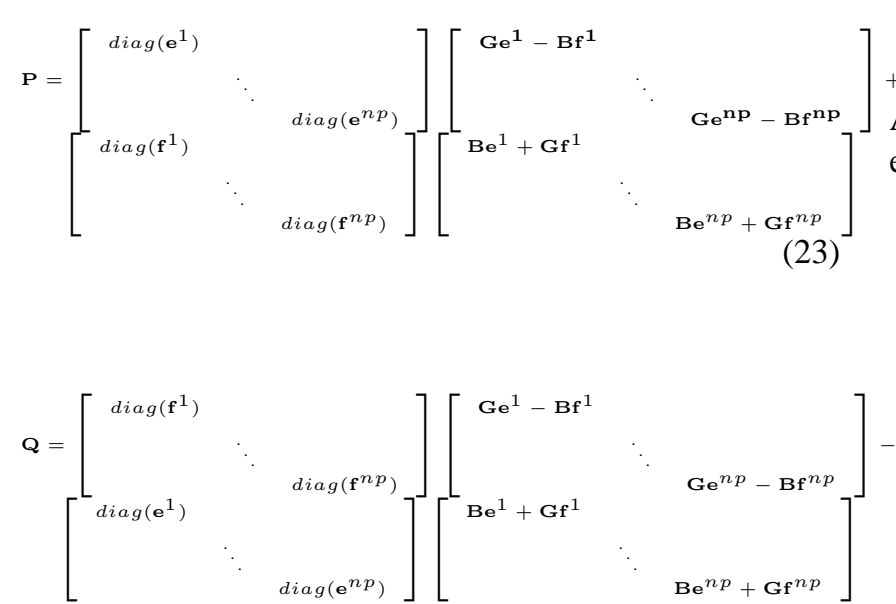

(24)

onde $\mathbf{e}^{k}=\left[\begin{array}{c}e_{1}^{k} \\ \vdots \\ e_{n b}^{k}\end{array}\right]:$ parte real da tensão no período $k, \mathbf{f}^{k}=$ $\left[\begin{array}{c}f_{1}^{k} \\ \vdots \\ f_{n b}^{k}\end{array}\right]$ : parte imaginária da tensão no período $k$,

G: parte real de $\dot{\mathbf{Y}}$, ou seja, matriz de condutância de barra,
B: parte imaginária de $\dot{\mathbf{Y}}$, ou seja, matriz de susceptância de barra.

O fasor tensão pode ser expresso, de forma compacta, em função de um vetor $\mathbf{x}$, cujos elementos são as componentes reais e imaginárias dos fasores de tensões nas barras:

$$
\mathbf{x}=\left[\begin{array}{llllll}
\left(\mathbf{e}^{1}\right)^{T} & \ldots & \left(\mathbf{e}^{n p}\right)^{T} \ldots & \left(\mathbf{f}^{1}\right)^{T} & \ldots & \left(\mathbf{f}^{n p}\right)^{T}
\end{array}\right]^{T}
$$

Para facilitar os cálculos e se obter os vetores e e f a partir do vetor $\mathbf{x}$ utilizam-se as seguintes matrizes:

$$
\begin{gathered}
\boldsymbol{\Gamma}_{e}=\left[\begin{array}{ll}
\boldsymbol{\Gamma} & \mathrm{N}
\end{array}\right] \\
\boldsymbol{\Gamma}_{\mathrm{f}}=\left[\begin{array}{lll}
\mathrm{N} & \boldsymbol{\Gamma}
\end{array}\right]
\end{gathered}
$$

onde

$\Gamma$ : matriz identidade de dimensão [(nb.np) $\times(n b . n p)]$;

$\mathbf{N}$ :matriz nula de dimensão $[(n b . n p) \times(n b . n p)] ; \boldsymbol{\Gamma}_{e}$ matriz composta pela justaposição da matriz $\Gamma$ e da matriz $\mathbf{N} \operatorname{logo}$ com dimensão $[(n b . n p) \times(2 . n b . n p)]$.

Assim, o vetor formado pelas partes reais (e) e pelas partes imaginárias (f) das tensões das barras pode ser escrito por:

$$
\mathbf{e}+j \cdot \mathbf{f}=\boldsymbol{\Gamma}_{e} \cdot \mathbf{x}+j \cdot \boldsymbol{\Gamma}_{f} \cdot \mathbf{x}
$$

Ainda, para que se possam equacionar convenientemente as equações (23) e (24) é preciso definir as seguintes matrizes:

$$
\begin{aligned}
& G G=\left[\begin{array}{lll}
\mathbf{G} & & \\
& \ddots & \\
& & \mathbf{G}
\end{array}\right] \\
& B B=\left[\begin{array}{lll}
\mathbf{B} & & \\
& \ddots & \\
& & \mathbf{B}
\end{array}\right]
\end{aligned}
$$

onde $G G$ : matriz de matrizes $\mathbf{G}$ dispostas diagonalmente com dimensão $[n b . n p \times n b . n p] ; B B$ : matriz de matrizes $\mathbf{B}$ dispostas diagonalmente com dimensão $[n b . n p \times n b . n p]$.

Utilizando-se as matrizes descritas em (26) e (27), pode-se representar as equações em função das variáveis $\mathbf{P g}, \mathbf{Q g}$ e $\mathbf{x}$.

$$
\begin{aligned}
\mathbf{P g}-\mathbf{P d} & =\left\{\operatorname{diag}\left(\boldsymbol{\Gamma}_{\mathbf{e}} \cdot \mathbf{x}\right) \cdot[\mathbf{G G}-\mathbf{B B}]+\right. \\
& \left.+\operatorname{diag}\left(\boldsymbol{\Gamma}_{\mathbf{f}} \cdot \mathbf{x}\right) \cdot[\mathbf{B B}-\mathbf{G G}]\right\} \cdot \mathbf{x}
\end{aligned}
$$




$$
\begin{aligned}
\mathbf{Q g}-\mathbf{Q d} & =\left\{\operatorname{diag}\left(\boldsymbol{\Gamma}_{\mathbf{f}} \cdot \mathbf{x}\right) \cdot[\mathbf{G G}-\mathbf{B B}]+\right. \\
& \left.+\operatorname{diag}\left(\boldsymbol{\Gamma}_{\mathbf{e}} \cdot \mathbf{x}\right) \cdot[\mathbf{B B}-\mathbf{G G}]\right\} \cdot \mathbf{x}
\end{aligned}
$$

Neste ponto, observa-se que as equações (31) e (32) representadas na forma retangular são funções quadráticas.

\subsection{Estabelecimento de Ângulo de Refe- rência}

Deve-se considerar uma referência angular. Como a representação escolhida para o fasor tensão é a retangular, esta referência implica que a parte imaginária do valor de tensão na forma retangular na barra de referência (ref), para cada período, é igual a zero:

$$
f_{r e f}^{k}=0 \quad k=1, \ldots, n p
$$

Essa consideração pode ser modelada utilizando uma matriz d de dimensão $[n p \times(2 . n b . n p)]$, que permite estabelecer matricialmente a equação (25). Deste modo:

$$
\mathbf{d} \cdot \mathbf{x}=\mathbf{0}
$$

\subsection{Restrições de Desigualdade}

Os limites operacionais dos geradores são:

$$
\begin{gathered}
\text { Phmin } \leq \mathbf{P g h} \leq \text { Phmax } \\
\text { Qhmin } \leq \text { Qgh } \leq \text { Qhmax } \\
\text { Ptmin } \leq \text { Pgt } \leq \text { Ptmax } \\
\text { Qtmin } \leq \text { Qgt } \leq \text { Qtmax }
\end{gathered}
$$

Como se trabalha com o fasor de tensão na forma retangular, é preciso que se faça uma adequada representação dos módulos de tensão ao quadrado:

$$
\left(\operatorname{Vmin}_{i}^{k}\right)^{2} \leq \mathbf{x}^{T} \cdot \mathbf{M}_{i}^{k} \cdot \mathbf{x} \leq\left(\operatorname{Vmax}_{i}^{k}\right)^{2} \quad i=1, \ldots n b
$$

onde $\left(V \min _{i}^{k}\right)^{2}$ e $\left(V \max _{i}^{k}\right)^{2}$ correspondem aos valores mínimo e máximo das magnitude de tensão permitidos na barra $i$ e período $k$, e, $\mathbf{M}_{i}^{k}$ matriz auxiliar de dimensão [2.nb.np $\times$ 2.nb.np] usada para obtenção da soma ao quadrado das partes reais e imaginárias de cada componente de tensão na barra $i$ e período $k$, detalhada no Anexo A.
O despacho das geradoras hidráulicas deve satisfazer os valores de metas energéticas Meta $_{i}$ para cada usina hidráulica, $i$, os quais são estabelecidos pelo planejamento de médio-prazo para todo período em estudo:

$$
\sum_{k=1}^{n p} P g h_{i}^{k} \leq \text { Meta }_{i} \quad i=1, \ldots, n g h
$$

onde $n g h$ é o número de geradores hidráulicos.

A reserva de potência representa uma folga de capacidade de geração, necessária para permitir que se mantenha a qualidade de suprimento na ocorrência de manutenções programadas e falhas das unidades geradoras, erros de previsão de carga e necessidade de regulação de freqüência do sistema. Ela é modelada como sendo a diferença entre a produção máxima e a atual de um determinado subconjunto de usinas (Provençano, 2003), e deve ser maior ou igual a um valor pré-especificado, ou seja:

$$
\begin{array}{r}
\sum_{i \in I}\left[\left(P g h_{\max i}+P g t_{\max i}\right)-\left(P g h_{i}^{k}+P g h_{i}^{k}\right)\right]>R^{k} \\
k=1, \ldots, n p
\end{array}
$$

onde $\mathbf{I}$ é conjunto de usinas associadas à reserva girante $\mathrm{e}$ $R^{k}$ é o valor de reserva girante para o subconjunto de usinas especificadas para o período $k$ e se refere a uma percentagem da carga total a cada período.

Como o problema tem grandes proporções, a monitoração dos limites de fluxo de potência ativa completo requer muito esforço computacional. Assim, adotou-se a formulação do fluxo de potência linearizado, simplificando a monitoração dessa grandeza (Provençano, 2003):

$$
\mathbf{F l}_{\mathbf{c c}}{ }^{k}=\mathbf{M}_{\mathbf{F L}} \cdot \mathbf{P}^{k} \quad k=1, \ldots, n p
$$

onde $\mathbf{M}_{\mathrm{FL}}$ é uma matriz auxiliar para o cálculo do fluxo linearizado nas linhas para o período $k, \mathbf{P}^{k}$ é o vetor de injeção de potência para o período $k$ e $\mathbf{F l}_{\mathbf{c c}}{ }^{k}$ vetor de fluxo de potência ativa circulante pelos ramos da rede para o período $k$. A descrição da matriz $\mathbf{M}_{\mathrm{FL}}$ está apresentada no Anexo B.

Assim, a restrição operacional de limite de fluxo nas linhas é expressa como:

$$
\boldsymbol{- F \operatorname { m a x }} \leq \mathbf{M}_{\mathbf{F L}} \cdot \mathbf{P}^{k} \leq \mathbf{F m a x} \quad k=1, \ldots, n p
$$

onde Fmax: limites de fluxos de potência ativa circulantes pelas linhas de transmissão.

Nos modelos de PD até aqui estudados a restrição de fluxo de potência ativa consiste na incorporação de duas restrições 
(limite máximo de fluxo e limite mínimo de fluxo) para cada linha, tal abordagem devido à grande quantidade de linhas e grande número de períodos considerados no $\mathrm{PD}$, torna a restrição de fluxo muito custosa computacionalmente.

A fim de contornar essa questão, ou seja, para um melhor desempenho computacional, foi adotada uma estratégia, similar à utilizada em CEPEL (2003), para consideração dos limites de transmissão, tal estratégia é baseada em dois Prédespachos complementares, conforme a Figura 1.

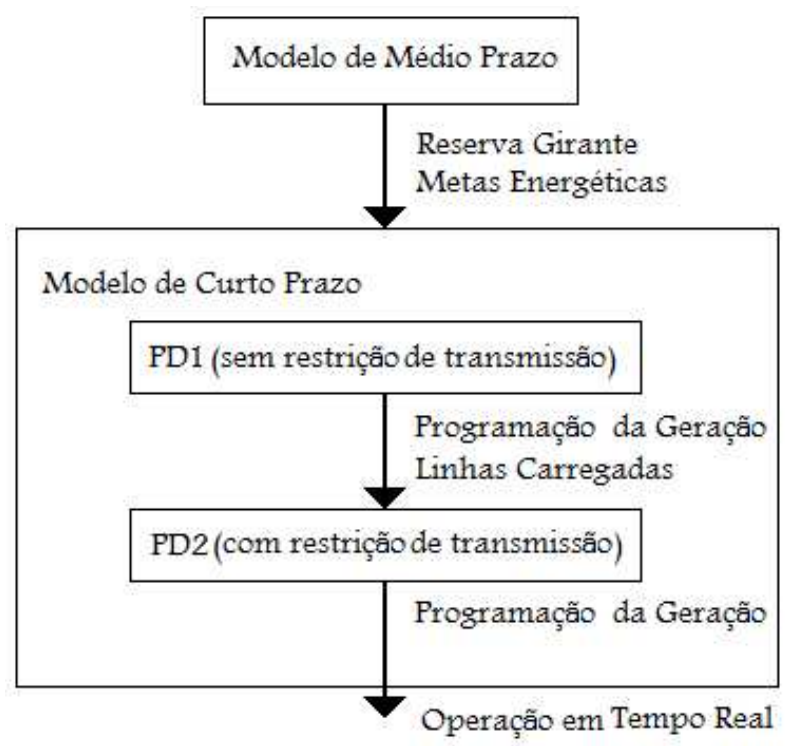

Figura 1: Estratégia para Consideração do Limite de Transmissão.

Primeiramente, resolve-se o Pré-Despacho denominado "PD1", desconsiderando os limites de fluxos ativos nas linhas de transmissão. Após a convergência, é realizado o cálculo dos fluxos de potência ativa entre as barras (linhas de transmissão) em todos os períodos, encontrando assim a magnitude e sentido do fluxo de potência ativa em todas as linhas de transmissão em todos os períodos. Em seguida, é realizada uma varredura de quais linhas se encontram sobrecarregadas.

A partir da solução do PD1 e do conhecimento de quais linhas se encontram carregadas, um novo Pré Despacho denominado, "PD2", é realizado, considerando agora os limites de fluxo somente nas linhas de transmissão que se encontram sobrecarregadas. Essa etapa do PD2 deve ser repetida iterativamente acrescentando-se restrições violadas até que não ocorram mais violações (Diniz et alli, 2002).

O problema de otimização completo é, então, o seguinte:

$$
\text { f.o. }=w c \cdot \mathbf{c}(\mathbf{P g t})+w p \cdot \sum(\mathbf{P g t}+\mathbf{P g h})
$$

$$
\text { s.a } \mathbf{P g}-\mathbf{P d}=\mathbf{P}(\mathbf{x})
$$

$$
\mathbf{Q g}-\mathbf{Q d}=\mathbf{Q}(\mathbf{x})
$$

$$
\text { Phmin } \leq \text { Pgh } \leq \text { Phmax }
$$

$$
\text { Qhmin } \leq \text { Qgh } \leq \text { Qhmax }
$$

$$
\mathbf{P t m i n} \leq \mathbf{P g t} \leq \mathbf{P t m a x}
$$

$$
\text { Qtmin } \leq \text { Qgt } \leq \text { Qtmax }
$$

$$
\begin{gathered}
\left(V \min _{i}^{k}\right)^{2} \leq \mathbf{x}^{T} \cdot \mathbf{M}_{i}^{k} \cdot \mathbf{x} \leq\left(\operatorname{Vmax}{ }_{i}^{k}\right)^{2} \quad i=1, \ldots n b \\
\sum_{k=1}^{n p} \operatorname{Pgh}_{i}^{k} \leq \text { Meta }_{i} \quad i=1, \ldots, n g h
\end{gathered}
$$

$$
\begin{array}{r}
\sum_{i \in I}\left[\left(P g h_{\max i}+P g t_{\max i}\right)-\left(P g h_{i}^{k}+P g h_{i}^{k}\right)\right]>R^{k} \\
k=1, \ldots, n p
\end{array}
$$

$$
-\mathbf{F m a x} \leq \mathbf{M}_{\mathbf{F L}} \cdot \mathbf{P}^{k} \leq \mathbf{F} \max \quad k=1, \ldots, n p
$$

Esse problema é resolvido pelo Método dos Pontos Interiores versão Primal-Dual (Granville, 1994). Esse método obtém a solução ótima mantendo a direção de busca dentro da região delimitada pelas restrições. Para tanto, transforma as inequações do problema em restrições de igualdade através da introdução de variáveis de folga (s) e considera a nãonegatividade das mesmas através da adição de uma função barreira logarítmica na função objetivo $(\mu \cdot \ln (\mathrm{s}))$. O parâmetro barreira, $\mu$, deve ser atualizado a cada iteração até que o mesmo se anule se deixe de perturbar a função objetivo até o final do processo de convergência.

A atualização do parâmetro barreira logarítmica $\mu$ é baseado no decréscimo do gap de dualidade $\left(\mathbf{s}^{T} \cdot \pi\right)$ :

$$
\mu=\frac{\mathbf{s}^{T} \cdot \pi}{2 \cdot n \cdot \beta}
$$


onde $n$ : número total de variáveis primais e duais; $\beta$ : fator de aceleração ( $\geq 1)$; $\pi$ : multiplicador de Lagrange relacionado às restrições de desigualdade.

A fim de diminuir a dimensão do sistema não linear resolvido e reduzir o tempo computacional de cada iteração, providenciou-se uma redução do mesmo, sendo que se mantiveram as variáveis primais (Pgt, Pgh e x) e os multiplicadores de Lagrange relacionados às restrições de igualdade (46) e (47). As variáveis de folga e os demais multiplicadores de Lagrange são calculados após a resolução do sistema linear reduzido.

\section{RESULTADOS}

Os resultados fornecidos pela formulação proposta são obtidos para dois sistemas, de 5 e 291 barras (Borges, 2010), considerados de pequeno e médio porte. Os resultados em pu estão na base 100MVA.

As simulações foram executadas através de um processador Intel (R) CORE ${ }^{\mathrm{TM}}$ i5, CPU 650, 3,20 Ghz, 4GB. E, foi usado o Matlab Versão R2006a como plataforma.

\subsection{Sistema de 5 barras}

O diagrama unifilar do sistema de 5 barras encontra-se na Figura 2.

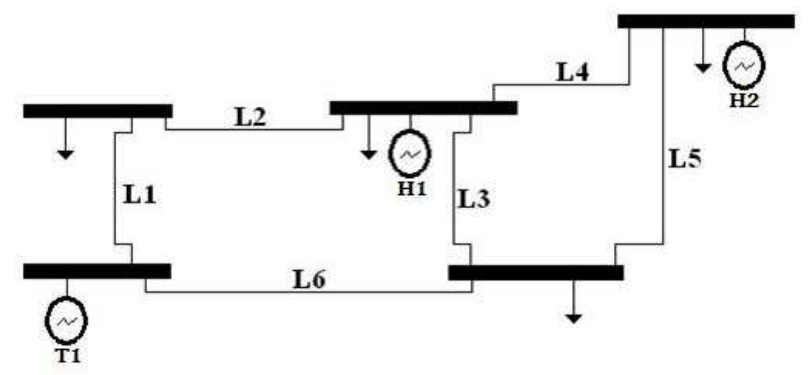

Figura 2: Sistema 5 barras

Para análise dos resultados numéricos foram escolhidos 3 cenários diferentes:

- Primeiro Cenário: caso base que serve de referência;

- Segundo Cenário: redução da Meta Energética para o gerador hidráulico 1 ;

- Terceiro Cenário: aumento da Reserva Girante.

Foram consideradas algumas combinações de valores para os pesos $w c$ e $w p$, tais como: $w c=1$ e $w p=10 ; w c=10$ e $w p=1$; $w c=1$ e $w p=1$. Para essas combinações, os despachos obtidos para os três cenários citados foram iguais. Assim, adotou-se $w c=1$ e $w p=1$.

Cenário 1: O pré-despacho é apresentado na Figura 3, cuja abscissa representa cada um dos 24 períodos e a ordenada os valores de despacho de geração ativa para cada uma das máquinas hidráulicas e térmicas bem como o valor total de carga ativa.

Já a Figura 4 apresenta o comportamento do perfil de da magnitude de tensão de cada barra a cada período.

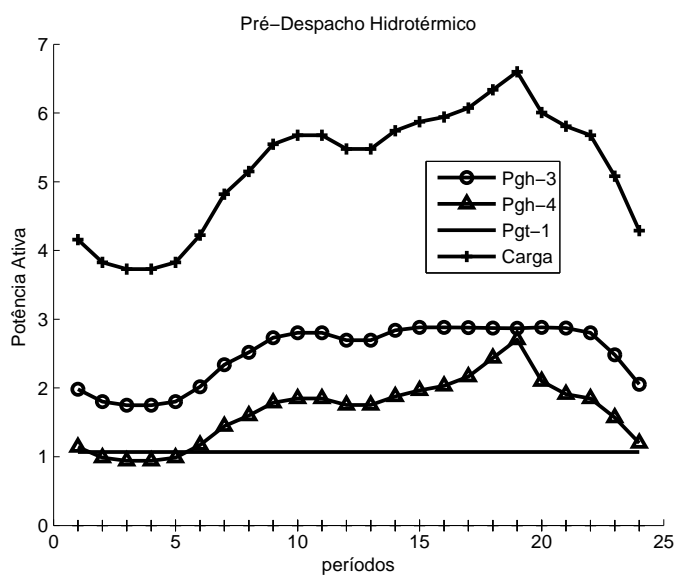

Figura 3: Despacho de geração - Caso Base.

Segundo a Figura 3, observa-se que os limites de geração térmica e hidráulica estão satisfeito (segundo dados contidos no Anexo A) ao longo dos 24 períodos.

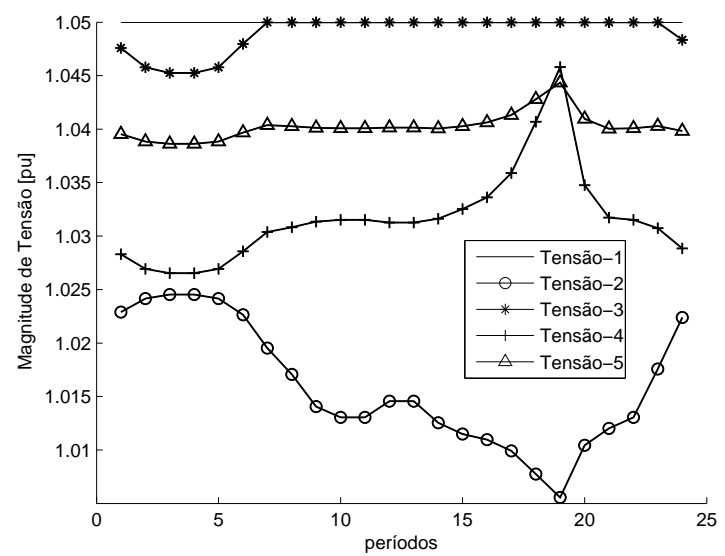

Figura 4: Perfil de Tensão - Caso Base.

Segundo a Figura 4, observa-se que os limites máximos de tensão foram atingidos na barra de geração térmica a partir 
Tabela 1: Fluxo de Potência Ótimo para o período 19 (Carga pesada)

\begin{tabular}{|c|c|c|c|c|c|c|c|c|}
\hline Barra & Tensão[pu] & $\hat{A ̂ n g}_{\text {.[graus] }}$ & $\mathrm{Pgt}^{19}{ }_{\text {[pu] }}$ & $\mathrm{Qgt}^{19}{ }_{\text {[pu] }}$ & $\mathrm{Pgh}^{19}{ }_{\text {[pu] }}$ & $\mathrm{Qgh}^{19}{ }_{\text {[pu] }}$ & $\mathrm{Pd}^{19}{ }_{\text {[pu] }}$ & $\mathrm{Qd}^{19}{ }_{\text {[pu] }}$ \\
\hline 1 & 1,0500 & 0 & 1,0700 & 0,0840 & 0 & 0 & 0 & 0 \\
\hline 2 & 1,0056 & $-6,5888$ & 0 & 0 & 0 & 0 & 1,5 & 0,2 \\
\hline 3 & 1,0500 & $-1,5519$ & 0 & 0 & 2,8680 & 0,2044 & 2 & 0 \\
\hline 4 & 1,0458 & $-1,9642$ & 0 & 0 & 2,7060 & 0,0835 & 2,7 & 0,1 \\
\hline 5 & 1,0443 & $-2,1221$ & 0 & 0 & 0 & 0 & 0,4 & 0 \\
\hline
\end{tabular}

do sétimo até o vigésimo terceiro período. A barra de carga 2 atinge valor mínimo de tensão no período referente ao maior carregamento.

Escolheu-se o período 19 (dentro de intervalo de carga pesada) para que se possa analisar em detalhes o comportamento da metodologia proposta. A Tabela 1 apresenta o despacho final para esse período. Ela inclui os valores de despacho de geração ativa e reativa para os geradores térmicos e hidráulicos e carga ativa e reativa de cada barra. A Tabela 2 apresenta o balanço total para todos os 24 períodos.

Tabela 2: Balanço de Potência Ativa para Pré-Despacho Cenário 1

\begin{tabular}{|c|c|c|c|c|}
\hline$\sum_{\text {letricalpul }}$ Pgh Hidre- & $\sum_{\text {micalpu] }}$ Pgt Tér- & $\sum_{\sum_{\mathrm{Pgt}[\mathrm{pu}]}^{\mathrm{pgh}}+}$ & $\sum_{\text {PdDemandalpu] }}$ & $\sum_{\text {Transmisssãofpuu }}$ Perdas- \\
\hline 100 & 25,6802 & 125,6802 & 124,7400 & 0,9402 \\
\hline
\end{tabular}

Cenário 2: A partir da do cenário 1, mas agora diminuindo a meta energética da usina hidráulica um, de 60 pu para 40 pu, chega-se ao pré-despacho apresentado na Figura 5, perfil de tensão na Figura 6 e balanços de potências na Tabela 3.

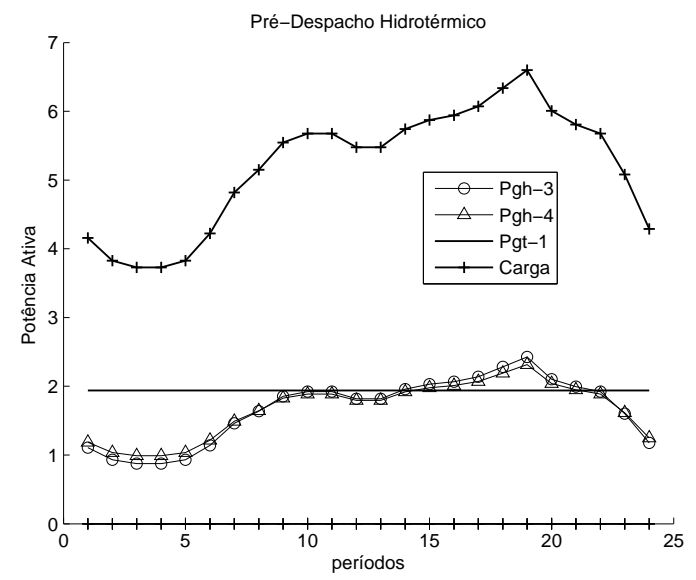

Figura 5: Despacho de Geração - Cenário 2.

Comparando os dados das tabelas 2 e 3, observa-se que a diminuição da meta energética de uma das usinas hidrelétricas,

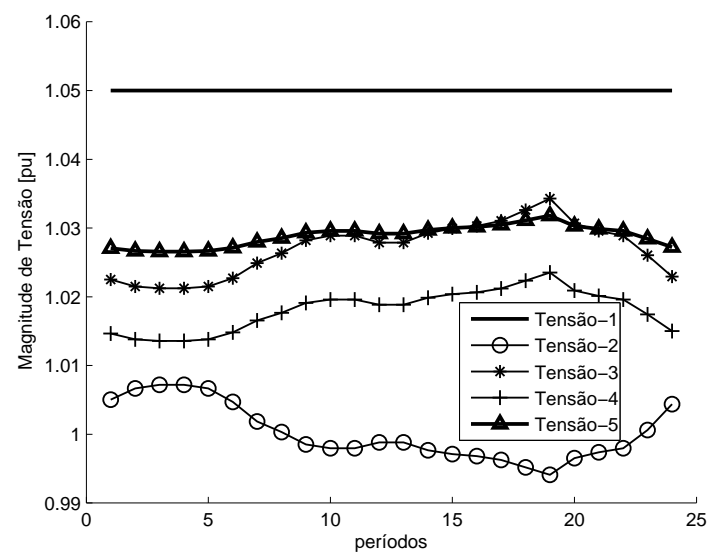

Figura 6: Perfil de Tensão - Cenário 2.

Tabela 3: Balanço de Potência Ativa para Pré-Despacho Cenário 2

\begin{tabular}{|c|c|c|c|c|}
\hline$\sum_{\text {Hidreletricalpul }}$ Pgh & $\sum_{\text {Térmicalpul }}$ Pgt & 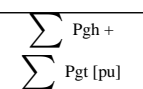 & $\sum_{\text {PdDemanda[pu] }}$ & $\sum_{\text {PerdasTransmissãofpu] }}$ \\
\hline 80 & 46,5567 & 126,5567 & 124,7400 & 1,8167 \\
\hline
\end{tabular}

resulta em diminuição da geração de potência ativa da usina hidrelétrica em questão. Tal configuração faz com que aumentem a geração de potência da usina térmica (atendimento a demanda), o custo com combustível e perdas elétricas.

O aumento das perdas se dá porque $70 \%$ da carga total estão instaladas nas barras 3 e 4 que também são barras de geração hidráulica. Ao se deslocar parte da geração para a térmica, passa-se, naturalmente, a se utilizar mais da malha de transmissão, provocando um aumento expressivo das perdas.

Outro ponto que merece destaque é a proximidade entre a curva de despacho da usina hidrelétrica um e da usina hidrelétrica dois, tal fato ocorre por que as metas de geração para as duas usinas neste cenário são iguais.

Cenário 3: Partindo da mesma configuração do cenário 1 , mas agora aumentando o valor da reserva girante de $2 \%$ para 
$6 \%$ da carga total por período, chega-se ao pré-despacho apresentado na Figura 7, perfil de tensão na Figura 8 e balanços de potências na Tabela 4.

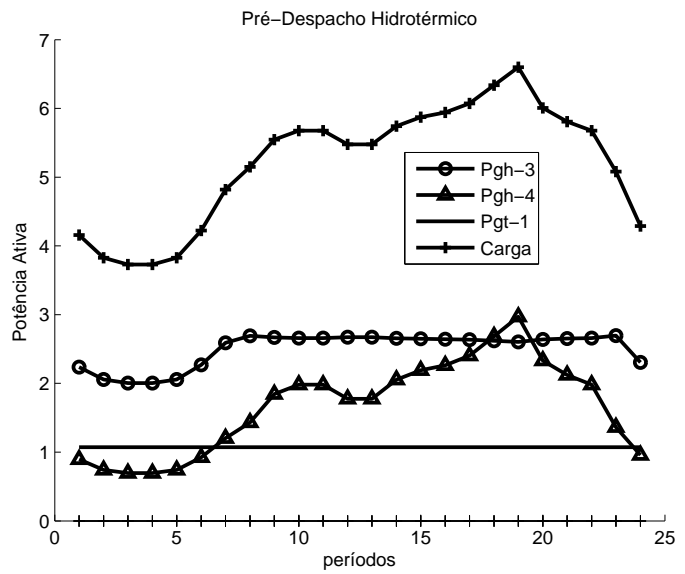

Figura 7: Despacho de Geração - Aumento da Reserva Girante.

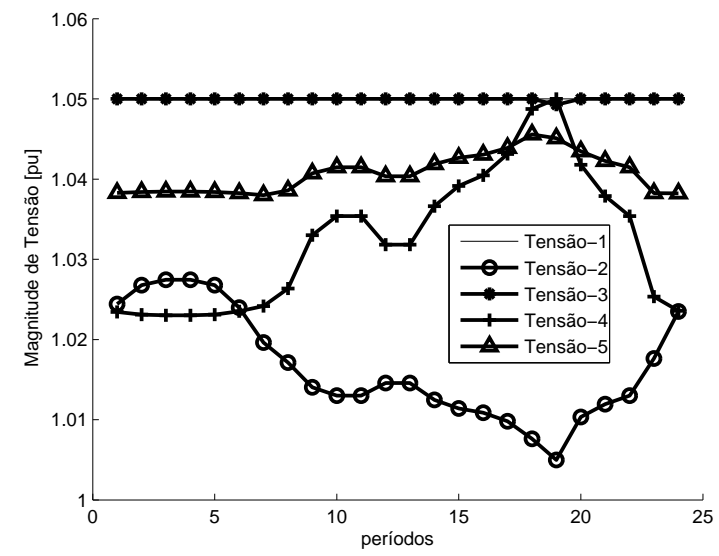

Figura 8: Perfil de Tensão - Aumento da Reserva Girante.

Comparando os resultados do cenário 1 e cenário 3, observase que o aumento da reserva girante alocada em uma das usinas hidrelétricas, resulta na diminuição da geração de potência da usina hidrelétrica no período de ponta de carga, e alocação da energia da usina no período de menor carga. Além disso, nota-se que, o aumento da restrição de reserva girante leva ao aumento da perda elétrica.

\subsection{Sistema de 291 barras}

O sistema de 291 barras (404 ramos) é um sistema reduzido proveniente do sistema elétrico do estado do Paraná e parte da região sul do sistema elétrico brasileiro. Ele possui 6 ge-
Tabela 4: Balanço de Potência Ativa para Pré-Despacho Cenário 3

\begin{tabular}{|l|l|l|l|l|}
\hline$\sum_{\substack{\text { Pgh Hi- } \\
\text { drelétricalpu] }}}$ & $\sum_{\text {mica[pu] }}$ Pgt Tér- & $\sum_{\text {Pgh }}+$ & $\sum_{\text {Pgt [pu] }}$ & $\sum_{\text {PdDemanda[pu] }}$ Perdas- \\
\hline 100 & 25,7428 & 125,7428 & 124,7400 & 1,0028 \\
\hline
\end{tabular}

radores térmicos com capacidade total instalada de $20 \mathrm{pu}$ e 28 geradores hidráulicos com capacidade total instalada de 62 pu. A carga de referência é de $(29,1+5,9$ j $)$ pu. A curva de carga diária considerada é a mesma que a utilizada pelo sistema de 5 barras (Anexo C).

Devido ao tamanho do sistema, optou-se por apresentar apenas dois cenários para o sistema de 291 barras, um cenário representando o período chuvoso, e outro representando o período seco:

- Cenário Úmido: período úmido (metas energéticas grandes para as usinas hidrelétricas totalizando 14.000 puh);

- Cenário Seco: período seco (metas energéticas pequenas para as usinas hidrelétricas totalizando 960 puh).

Cenário Úmido: Como o sistema de 291 barras tem muitas barras, apresentam-se na Figura 9, apenas o despacho das 3 maiores geradoras hidráulicas e 3 maiores geradoras térmicas e na Figura 10 o perfil e tensão dessas barras.

O despacho foi obtido usando três combinações de pesos para as funções objetivo: (i) $w c=10$ e $w p=1$; (ii) $w c=1 \mathrm{e}$ $w p=1$, e (iii) $w c=1$ e $w p=10$. Os resultados obtidos com a primeira combinação e segunda combinação são idênticos e é o que serão mostrados a seguir.

Na Figura 9, são representadas 2 usinas hidrelétricas: Pgh 24 corresponde ao despacho de 3 geradores da Usina Ney Braga, Pgh 111 e 113 correspondem ao despacho de 2 e 3 unidades geradoras da Usina Salto Osório, respectivamente. São também representadas três usinas térmicas: Pgt 3 corresponde ao despacho da usina Capivara, Pgt 4 corresponde ao despacho da usina Rosana e Pgt 5 corresponde ao despacho da usina Salto Grande. Salienta-se que essas usinas na realidade são hidráulicas e no estudo foram alocadas como térmicas, a fim de dar diversidade na análise.

Na Figura 10, são apresentados os despachos de potência reativa das usinas apresentadas na Figura 9, necessários para que se mantenham as magnitudes de tensão das barras dentro dos limites operacionais como apresentadas na Figura 11. $\mathrm{Na}$ Figura 10, observa-se que as gerações de potência reativa aumentam com o aumento da carga. Na Figura 11, observase que, para a minimização (44), todas as barras de geração, com exceção da barra 3, tem suas tensões aumentadas nos períodos de carga elevada. 


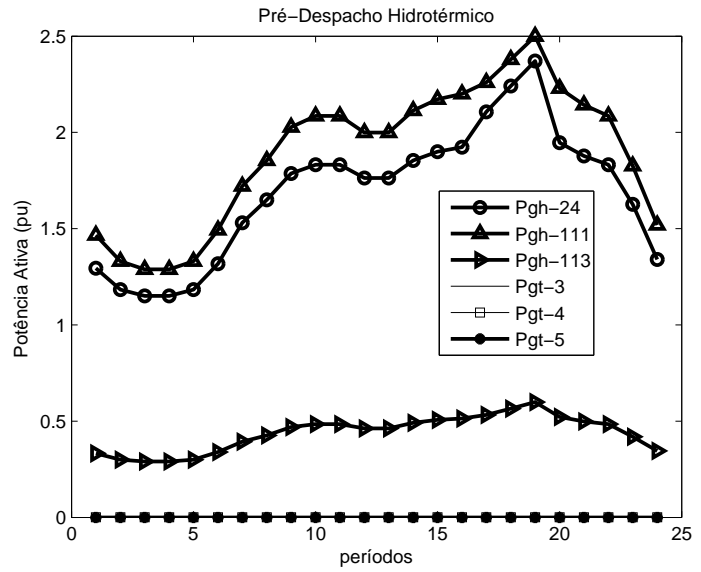

Figura 9: Despacho de Potência Ativa - Sistema de 291 barras - Período Úmido

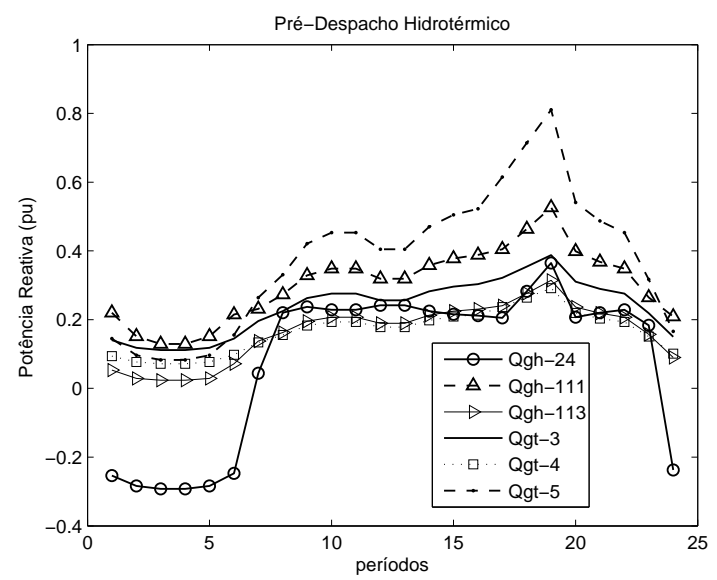

Figura 10: Despacho de Potência Reativa - Sistema de 291 barras - Período Úmido

Segundo a Tabela 5, que apresenta os despachos totais, observa-se que a geração das usinas termelétricas é zero. Tal fato ocorre porque as metas energéticas das usinas hidrelétricas são suficientes para suprir a demanda sem necessidade de complementação de energia por parte das usinas termelétricas.

No entanto, ao se utilizar os pesos $w c=1$ e $w p=10$, observa-se um despacho final diferenciado onde as perdas são menores e o custo das térmicas não é mais nulo, como mostrado na Tabela 6.

$(w c=1 \mathrm{e} w p=10)$

Como esse problema de otimização é multi-objetivo, não existe somente uma solução ótima para o problema e sim

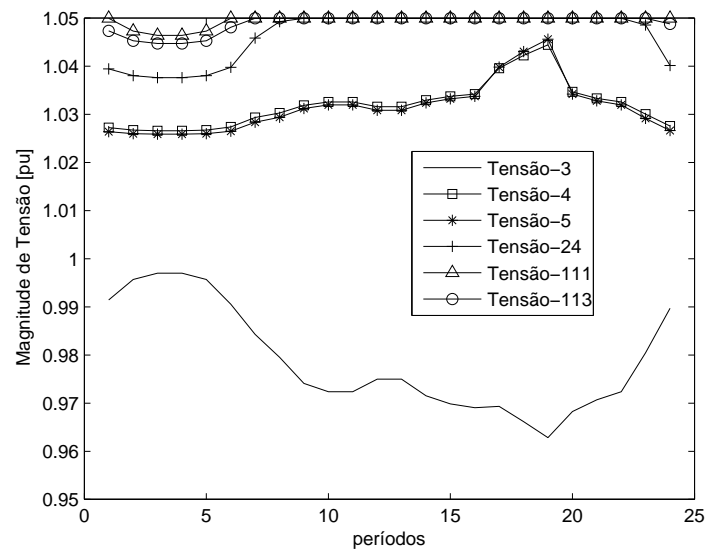

Figura 11: Perfil e Tensão - Sistema de 291 barras - Período Úmido

Tabela 5: Balanço de Potência Ativa para Pré-Despacho Úmido// $(w c=10$ e $w p=1 ; w c=1$ e $w p=1)$

\begin{tabular}{|c|c|c|c|c|}
\hline$\sum_{\text {létrica[pu] }}$ Pgh Hidre- & $\sum_{\text {Tér- }}$ Pgt & $\sum_{[\mathrm{pu}]}^{\mathrm{Pgh}+}$ & $\sum_{\text {manda[pu] }}$ PdDe- & $\sum_{\text {Transmissão[pu] }}$ Perdas- \\
\hline 559,8129 & 0 & 559,8129 & 549,8587 & 9,9542 \\
\hline
\end{tabular}

um conjunto de possíveis soluções eficientes (Pareto-ótimo), influenciadas pelos pesos considerados para cada um dos critérios de otimização, cabendo ao planejador a escolha dos mesmos de modo a enfatizar os critérios de seu interesse.

Assim, adotando-se a premissa que a minimização do custo das térmicas deve se enfatizado, optou-se pela combinação de pesos $w c=10$ e $w p=1$ (tanto no período úmido quanto no seco).

Cenário Seco: As Figuras 12 e 13 apresentam os despachos de potência ativa e reativa, respectivamente, das 5 usinas já analisadas no período úmido, e a Figura 14 as magnitudes de tensão dessas barras dentro dos limites operacionais. A Tabela 6 apresenta os despachos totais de potência ativa para esse cenário.

Segundo a Tabela 8, a geração das usinas termelétricas é diferente de zero no período seco. Tal fato ocorre porque as

Tabela 6: Balanço de Potência Ativa para Pré-Despacho Úmido (wc = 1 e wp=10)

\begin{tabular}{|c|c|c|c|c|}
\hline$\sum_{\text {létrica[pu] }}$ Pgh Hidre- & $\begin{array}{l}\sum_{\text {Tér- }} \text { Pgt } \\
\text { mica[pu] }\end{array}$ & $\sum_{[\mathrm{pu}]} \mathrm{Pgh}+$ & $\sum_{\text {manda[pu] }} \mathrm{PdDe}-$ & $\sum_{\text {Transmissão[pu] }}$ Perdas- \\
\hline 555,7 & 3,42480 & 559,1433 & 549,8587 & 9,2846 \\
\hline
\end{tabular}




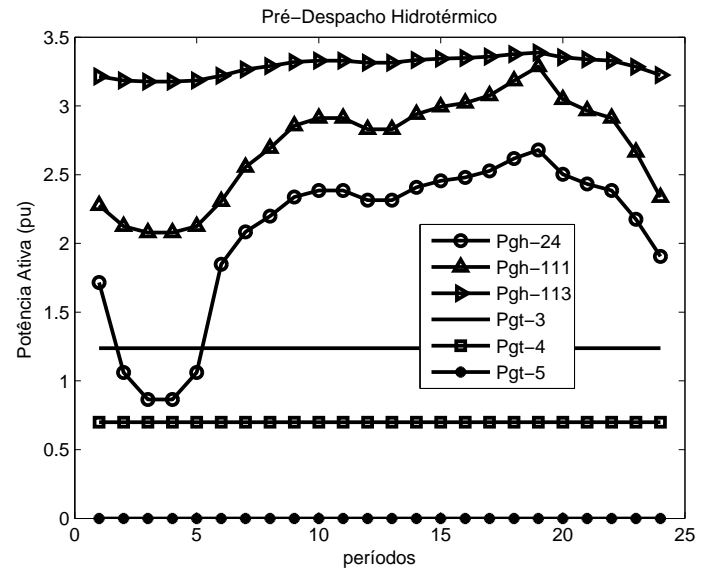

Figura 12: Despacho de Potência Ativa - Sistema de 291 barras - Período Seco

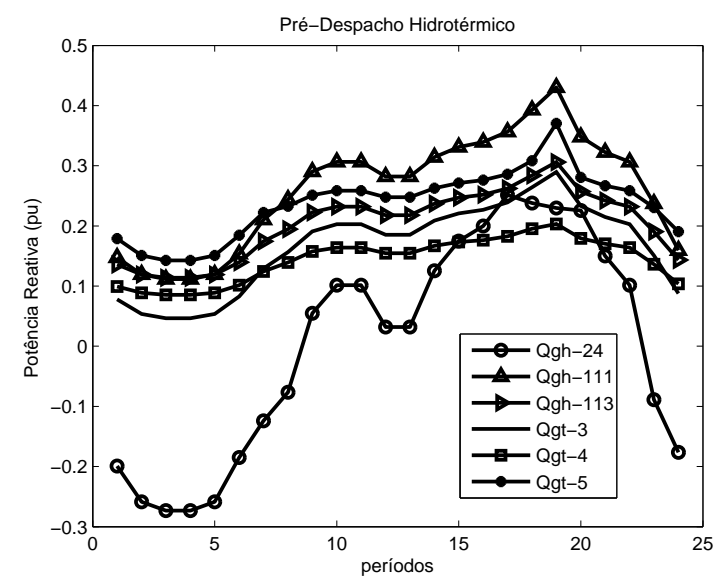

Figura 13: Despacho de Potência Reativa - Sistema de 291 barras - Período Seco

metas energéticas das usinas hidrelétricas não são suficientes para suprir a necessidade energética das cargas, havendo necessidade de complementação energética que é realizada através das usinas termelétricas.

Para confirmação do resultado do cenário seco, foi simulada a retirada das usinas termelétricas do sistema. Em tal simulação o algoritmo não atingiu convergência, evidenciando assim a necessidade de complementação térmica, que é requerida nesse cenário.

Os dados dos experimentos expressos em figuras e tabelas ilustram através de comparações de cenários, que a metodologia convergiu de maneira adequada respeitando as restrições impostas (limites de tensão e transmissão) e dados de entrada (meta energética e reserva de potência).

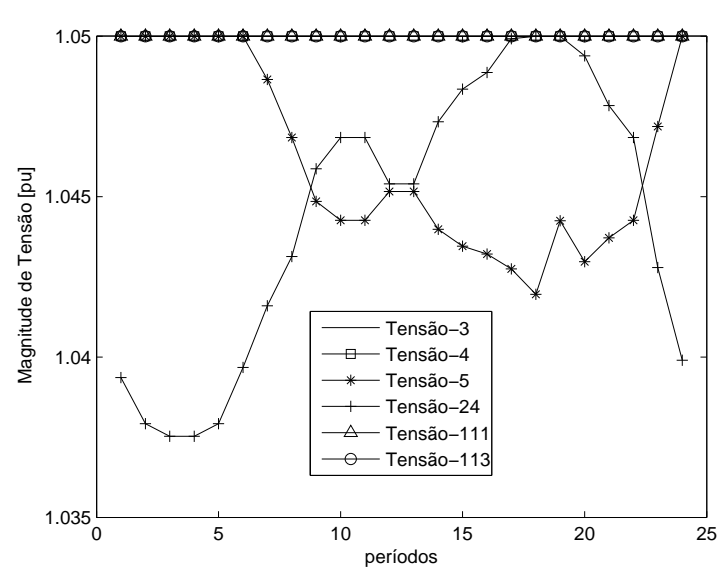

Figura 14: Perfil de Tensão - Sistema de 291 barras - Período Seco

Tabela 7: Balanço de Potência Ativa para Pré-Despacho Seco

\begin{tabular}{|c|c|c|c|c|}
\hline$\sum_{\text {létrica[pu] }}$ Pgh Hidre- & $\sum_{\substack{\text { Tér- } \\
\text { mica[pu] }}}$ & $\sum_{[\mathrm{pu}]} \mathrm{Pgh}+$ & $\sum_{\text {manda[pu] }}$ PdDe- & $\sum_{\text {Transmissão[pu] }}$ Perdas- \\
\hline 411,2 & 146,5533 & 557,8269 & 549,8587 & 7,9542 \\
\hline
\end{tabular}

Há algumas peculiaridades do Método de Pontos Interiores utilizado que são:

- o número de iterações para convergência do problema depende do valor inicial dado ao parâmetro barreira, sendo que o valor ideal para $\mu$ inicial depende do sistema.

- a escolha do fator de aceleração $\beta$, utilizado para a atualização do parâmetro barreira, principalmente para sistemas mal-condicionados, é crucial para a convergência do algoritmo.

Assim, a Tabela 8 apresenta a influência dos parâmetros $\mu^{o}$ e $\beta$ no número de iterações. Foram feitas simulações para várias combinações de valores para $\mu^{o}$ e $\beta$.

Assim recomenda-se para o caso de 291 barras, recomendase o valor de $\mu^{o}=0,01$ e $\beta$ igual a 1 .

Para o período seco programa computacional convergiu em 32 iterações e em 200,53 s.

Afim melhorar o tempo computacional despendido pela metodologia, pode-se utilizar a versão Preditor-Corretor do Método dos Pontos Interiores e linguagens compiladas. Nesse trabalho foi utilizada a versão Primal-Dual Puro do MPI. 


\section{CONCLUSÃO}

Neste artigo, o problema de pré-despacho de sistema hidrotérmico foi modelado de modo a obter a geração de potência ativa e reativa de cada usina do sistema, minimizando as perdas elétricas e o custo operacional (combustível elétrica), com as seguintes considerações: planejamento diário (24 horas) de geração de potência ativa e reativa; utilização de metas energéticas obtidas por modelo de médio prazo; restrições hidráulicas ignoradas; despacho das térmicas realizado de forma constante ao longo do dia; utilização de limites de fluxo nas linhas de transmissão e reserva girante.

Todas essas considerações foram testadas através de diversos cenários, apresentando resultados satisfatórios.

Uma das principais vantagens dessa modelagem é a consideração da parte reativa, que permite um controle efetivo do perfil de tensão nas barras, que é um dos grandes gargalos da rede de transmissão e a consideração da forma retangular para representação do fasor tensão, que transforma as restrições de balanço de potência ativa e reativa em funções quadráticas de modo que a expansão em Série de Taylor é exata para o termo de ordem dois, o que contorna questões numéricas para o tratamento intertemporal do problema resolvido pelo Método dos Pontos Interiores.

\section{REFERÊNCIAS}

Almeida, K. C, Silva, A. S., Agostini, M. N., Silva, D. D., Soares, V. L. C. and Watanabe, E. H. (2009). Assessment of the Application of FACTS Equipment to the Brazilian Interconnected Power Systems. XI Simpósio de Especialistas em Planejamento da Operação e Expansão Elétrica (SEPOPE), IP-116.

Borges, S. S. (2010). Pré-Despacho de Potência Ativa e Reativa para Sistemas Hidrotérmicos Utilizando Método de Pontos Interiores e Coordenadas Retangulares. Dissertação de Mestrado, Universidade Federal do Paraná.

Tabela 8: Dados de Convergência - Úmido

\begin{tabular}{|l|l|l|l|}
\hline $\begin{array}{c}\text { Número de } \\
\text { Iterações }\end{array}$ & $\begin{array}{l}\text { Tempo até Conver- } \\
\text { gência [s] }\end{array}$ & $\begin{array}{l}\text { mi } \\
\text { inicial }\end{array}$ & Beta \\
\hline 28 & 167,31 & 0,01 & 12,5 \\
\hline 26 & 154,17 & 0,01 & 5 \\
\hline 21 & 120,77 & 0,01 & 1 \\
\hline 27 & 156,64 & 0,1 & 12,5 \\
\hline 25 & 146,08 & 0,1 & 5 \\
\hline 23 & 137,70 & 0,1 & 1 \\
\hline
\end{tabular}

CENTRO DE PESQUISAS DE ENERGIA ELETRICA (2003). Modelo DESSEM, versão $8.2 a$ - Relatório Técnico.

Carvalho, M. F. and Soares, S. (1987). An Efficient Hydrothermal Scheduling Algorithm. IEEE Transactions on Power Systems, Vol. 2, no 3, pp. 537-542.

Carvalho, M. F., Soares, S. and Ohishi, T. (1988). Optimal Active Power Dispatch by Network Flow Approach. IEEE Transactions on Power Systems, Vol. $3, \mathrm{n}^{\circ} 3$, pp. 1640-1647.

Conejo, A. J. and Redondo, N. J. (1999). Short-Term HidroThermal Coordination by Lagrangian Relaxation: Solution of the Dual Problem, IEEE Transactions on Power Systems, Vol. 14, $\mathrm{n}^{o} 1$, pp. 89-95.

Diniz, A. S. L., Souza, L. C. D., Romero, S. P., Costa F. S., Macieira, M. E. P., Sagastizabal, C. A., Belloni, S. S. (2002). Estratégia de Representação DC da Rede Elétrica no Modelo de Despacho da Operação Energétcia - DESSEM, VIII Simpósio de Especialistas em Planejamento da Operação e Expansão Elétrica (SEPOPE), IP-127.

Drake, J. H., Kirchmayer, L. K. and Mayal, R. B. (1962). Optimum Operation of a Hydrothermal System. AIEE Transactions on Power Apparatus and Systems, Vol. PAS-80, pp. 242-250.

Fernandes, T. S. P. (2004). Um Modelo de Despacho Ótimo de Potência para Sistemas Multi-Usuários, Tese de Doutorado, Universidade Federal de Santa Catarina.

Franco, P., Carvalho, M. F. and Soares, S. (1994). A Network Flow Model for Short-Term Hydro-dominated Hydrotermal Scheduling Problem. IEEE Transactions on Power Systems, Vol. 9, $\mathrm{n}^{o}$ 2, pp. 1016-1022.

Granville, S. (1994). Optimal Reactive Power Dispatch Through Interior Point Methods. IEEE Transactions on Power Systems, Vol. 9, ${ }^{o}$ 1, pp. 136-146.

Habibollahzadeh, H. and Bubenko, A. (1986). Application of Decomposition Techniques to Short Term Operation Planning of Hydrothermal Power Systems. IEEE Transactions on Power Systems, Vol. 1, $\mathrm{n}^{o}$ 1, pp. 41-47.

Luo, G. X., Habibollahzadeh, H. and Semlyen, A. (1989). Short Ter Hydrotermal Dispatch Details Model and Solutions. IEEE Transactions on Power Systems, Vol. 4, $\mathrm{n}^{\circ} 4$, pp. 1452-1460.

Lu, S.; Sun, C. and Lu, Z. (2010). An Improved QuantumBehaved Particle Swarm Optimization Method for Short-Term Combined Economic Emission Hydrothermal Scheduling. Energy Conversion and Management, Vol. 51, pp. 561-571. 
Nepomuceno, L.; Ohishi, T. and Soares, S. (2000). Uma Metodologia de Pré-Despacho AC com Base em um Modelo de FPO Newton; Revista SBA Controle \& Automação, Vol. 11, $\mathrm{n}^{\circ}$ 2, pp. 169-175.

Nepomuceno, L.; Soares, S.; Ohishi, T. and Oliveira, A. L. (2002). Um Modelo de Pré-Despacho Ativo/Reativo para Sistemas Hidrotérmicos Utilizando Relaxação Lagrangeana. XIV Congresso Brasileiro de Automática, $B A$, pp.1-6.

Ohishi, T. (1990). Planejamento da Operação de Curto Prazo de Sistemas Hidrotérmicos Predominantemente Hidráulicos. Tese de Doutorado, Unicamp.

Pereira, M. V. F. and Pinto, L. M. V. (1983). Application of Decomposition Techniques to the Mid and Short Term Scheduling of Hydrothermal Systems. IEEE Transactions on Power Apparatus and Systems, Vol. PAS-102, $\mathrm{n}^{\circ}$ 11, pp. 3611-3618.

Provençano, F. (2003). Despacho Econômico em Usinas Hidrelétricas. Dissertação de Mestrado, Unicamp.

Saga. T. N and Khaparde, S. A. (1978). An Application of a Direct Method to the Optimal Scheduling of Hydrothermal System'. EEE Transactions on Power Apparatus and Systems, Vol. PAS-97, $\mathrm{n}^{\circ}$. 3, pp. 977-983.

Sifuentes, W. S. and Vargas, A. (2007). Hydrothermal Scheduling Using Benders Decomposition: Accelerating Techniques. IEEE Transactions on Power Systems, Vol. 22, $\mathrm{n}^{\circ}$ 3, pp. 1351-1359.

Soares, S. and Salmazo, C. T. (1997). Minimum Loss Predispatch Model for Hydroelectric Systems. IEEE Transactions on Power Systems, Vol. 12, $\mathrm{n}^{\circ} 3$, pp. 1220-1228.

Thomaz, A. (2007). Método de Pontos Interiores Aplicado ao Fluxo de Potência Ótimo Utilizando Coordenadas Cartesianas. Tese de Doutorado, Unicamp.

Torres, G. L. and Quintana, V. H. (1998). An Interior-Point Method for Nonlinear Optimal Power Flow using Voltage Rectangular Coordinates. IEEE Transactions on Power Systems, Vol. 13, no 4, pp. 1211-1218.

Wei, H., Sasaki, H. and Kubokawa, J. (1998). A Decoupled Solution of Hydro-Thermal Optimal Power Flow Problem by Means of Interior Point Method and Network Programming. IEEE Transactions on Power Systems, Vol. 13, no 2, pp. 286-293.

Wood, A. and Wollenberg, B. (1998). Power Generation Operation and Control, John Wiley \& Sons, Inc, New York.

\section{ANEXO A}

Para uma determinada barra $i$ do período $k$, o módulo ao quadrado da tensão é:

$$
\left|\dot{V}_{i}\right|^{2}=\left(e_{i}\right)^{2}+\left(f_{i}\right)^{2}
$$

Para que essa equação possa ser generalizada em função do vetor $\mathbf{x}$, realizam-se as seguintes operações:

$$
\mathbf{v e}_{i}^{T} \cdot \mathbf{x}=e_{i}
$$

onde

$\mathbf{v e}_{i}^{T}$ : i-ésima linha da matriz $\Gamma_{e}$

Portanto,

$\left(\mathbf{v e}_{i}^{T} \cdot \mathbf{x}\right)^{T} \cdot\left(\mathbf{v} \mathbf{e}_{i}^{T} \cdot \mathbf{x}\right)=\left(\mathbf{x}^{T} \cdot \mathbf{v} \mathbf{e}_{i}\right) \cdot\left(\mathbf{v} \mathbf{e}_{i}^{T} \cdot \mathbf{x}\right)=\left(e_{i}\right)^{2}$

Além disso,

$$
\mathbf{v f}_{i}^{T} \cdot \mathbf{x}=f_{i}
$$

onde

$\mathbf{v f}_{i}^{T}$ : i-ésima linha da matriz $\Gamma_{f}$;

Portanto,

$\left(\mathbf{v f}_{i}^{T} \cdot \mathbf{x}\right)^{T} \cdot\left(\mathbf{v f}_{i}^{T} \cdot \mathbf{x}\right)=\left(\mathbf{x}^{T} \cdot \mathbf{v f}_{i}\right) \cdot\left(\mathbf{v f}_{i}^{T} \cdot \mathbf{x}\right)=\left(f_{i}\right)^{2}$

Somando as equações (A.3) e (A.5) tem-se:

$$
\mathbf{x}^{T} \cdot\left(\mathbf{v} \mathbf{e}_{i} \cdot \mathbf{v e}_{i}^{T}+\mathbf{v} \mathbf{f}_{i} \cdot \mathbf{v} \mathbf{f}_{i}^{T}\right) \mathbf{x}=\mathbf{x}^{T} \cdot \mathbf{M}_{i} \cdot \mathbf{x}
$$

onde

$\mathbf{M}_{i}=\mathbf{v e}_{i} \cdot \mathbf{v} \mathbf{e}_{i}^{T}+\mathbf{v} \mathbf{f}_{i} \cdot \mathbf{v} \mathbf{f}_{i}^{T}$ : matriz auxiliar de dimensão $[2 . n b . n p \times 2 . n b . n p]$,usada para obtenção da soma ao quadrado das partes reais e imaginárias de cada componente de tensão na barra $i$. 


\section{ANEXO B}

Utilizando a formulação do fluxo de potência linear, a definição de injeção de potência ativa é:

$$
\mathbf{P}^{k}=\beta \cdot \theta^{\mathbf{k}}
$$

onde

$\mathbf{P}^{k}=\beta$ : vetor composto pelos ângulos pelas injeções de potência no período $k$, sem a posição referente à barra de referência, com dimensão [(nb-1)x 1];

$\beta$ : matriz do tipo susceptância de barra sem a linha e coluna referente à barra de referência com dimensão $[(n b-1) \times(n b-$ $1)]$

$\theta^{\mathbf{k}}$ : vetor composto pelos ângulos das barras, $i$, no período $k$, com dimensão [(nb-1)x 1].

Ainda do modelo linearizado sabe-se que fluxo linear para cada linha do período $k$ é dado por:

$$
\mathbf{F l}^{k}=\mathbf{X}^{-1} \cdot \mathbf{A}^{k} \cdot \theta^{\mathbf{k}}
$$

onde

$\mathbf{F l}_{c c}^{k}$ : vetor de fluxo de potência através de $n l$ linhas para o período $k$, de dimensão [ $n l$ x 1$]$;

$\mathbf{A}^{k}$ : matriz de incidência ramo-linha para o período $k$, de dimensão $[n l \times(n b-1)]$.

Como

$$
\theta^{\mathbf{k}}=\left(\beta^{\prime}\right)^{-1} \cdot \mathbf{P}^{k}
$$

E, substituindo (B.3) em (B.2):

$$
\mathbf{F} \mathbf{l}^{k}=\mathbf{X}^{-1} \cdot \mathbf{A}^{k} \cdot\left(\beta^{\prime}\right)^{1} \cdot \mathbf{P}^{k}=\mathbf{M}_{\mathbf{F L}} \cdot \mathbf{P}^{k}
$$

\section{ANEXO C}

O limite de magnitude de tensão mínimo é igual a 0,95 pu e de tensão máximo é igual a 1,05 pu para todas as barras. Os limites mínimos de geração térmica e hidráulica são nulos.

Os valores de carga $\mathbf{P d}+\mathbf{j} \mathbf{Q d}$ são usados como carga básica. A curva de carga diária considerada é $63 \% ; 58 \% ; 56,5 \%$; 56,5\%; 58\%; 64\%; 73\%; 78\%; 84\%; 86\%; 86\%; 83\%; 83\%; $87 \%$; 89\%; 90\%; 92\%; 96\%; 100\%; 91\%; 88\%; 86\%; $77 \%$
Tabela C.1: Dados de Linha do Sistema de 5 barras

\begin{tabular}{|c|c|c|c|}
\hline Elemento & $\mathrm{r}(\mathrm{pu})$ & $\mathrm{x}(\mathrm{pu})$ & $\mathrm{b}^{\text {sh }}(\mathrm{pu})$ \\
\hline $1-2$ & 0,042 & 0,168 & 0,030 \\
\hline $2-3$ & 0,031 & 0,126 & 0,020 \\
\hline $3-5$ & 0,053 & 0,210 & 0,015 \\
\hline $3-4$ & 0,084 & 0,336 & 0,012 \\
\hline $5-4$ & 0,063 & 0,252 & 0,011 \\
\hline $5-1$ & 0,013 & 0,126 & 0,010 \\
\hline
\end{tabular}

Tabela C.2: Dados de Barra do Sistema de 5 Barras

\begin{tabular}{|c|c|c|c|c|c|c|}
\hline Bus & Pgtmax $_{[\mathrm{pu}]}$ & $\operatorname{Pghmax}_{[\mathrm{pu}]}$ & $\mathbf{a}_{[s / \mathrm{pu}]}$ & $\left.\mathbf{b}_{[\mathrm{s} / \mathrm{pu}}{ }^{2}\right]$ & $\mathbf{P d}_{[\mathrm{pu}]}$ & $\mathbf{Q d}_{[\mathrm{pu}]}$ \\
\hline 1 & 3 & 0 & 2 & 1 & 0 & 0 \\
\hline 2 & 0 & 0 & 0 & 0 & 1,5 & 0,2 \\
\hline 3 & 0 & 3 & 0 & 0 & 2,0 & 0 \\
\hline 4 & 0 & 3 & 0 & 0 & 2,7 & 0,1 \\
\hline 5 & 0 & 0 & 0 & 0 & 0,4 & 0 \\
\hline
\end{tabular}

e $65 \%$ da carga básica, para os períodos de 1 até 24 horas, respectivamente. 